\title{
On a type of almost Kenmotsu manifolds with harmonic curvature tensors
}

\author{
Yaning Wang $\quad$ Ximin Liu
}

\begin{abstract}
Let $M^{2 n+1}$ be an almost Kenmotsu manifold with the characteristic vector field belonging to the $(k, \mu)^{\prime}$-nullity distribution. We prove that the curvature tensor of $M^{2 n+1}$ is harmonic if and only if $M^{2 n+1}$ is locally isometric to either a product space $\mathbb{H}^{n+1}(-4) \times \mathbb{R}^{n}$, or an Einstein warped product $C \times{ }_{f} N^{2 n}$ of an open interval and a Ricci-flat almost Kähler manifold.
\end{abstract}

\section{Introduction}

A tensor field of type $(1,3)$ is called an algebraic curvature tensor field if it has symmetric properties of the curvature tensor field of a Riemannian manifold $(M, g)$. It is well known [17] that an algebraic curvature tensor field $R$ on a Riemannian manifold $(M, g)$ is said to be harmonic if

$$
(\operatorname{div} R)(X, Y, Z)=0
$$

for any vector fields $X, Y, Z$ on $M$, where div denotes the divergence operator with respect to the metric $g$. Following [16], an algebraic curvature tensor field satisfying the second Bianchi identity is harmonic if and only if the associated Ricci operator is of Codazzi type, that is,

$$
\left(\nabla_{X} Q\right) Y=\left(\nabla_{Y} Q\right) X
$$

Received by the editors in November 2013 - In revised form in May 2014.

Communicated by F. Bourgeois.

2010 Mathematics Subject Classification : 53C15, 53C25, 53D15.

Key words and phrases : almost Kenmotsu manifold, $(k, \mu)^{\prime}$-nullity distribution, harmonic curvature tensor, warped product. 
for any vector fields $X, Y$ on $M$. Obviously, by the above statements we see that the curvature tensor of a Ricci symmetric Riemannian manifold (i.e., $\nabla Q=0$ ) is harmonic. One important reason for the interest in studying the Riemannian manifolds with harmonic curvature tensors lies in the fact that, the curvature tensor of a Riemannian manifold $(M, g)$ is harmonic if and only if the Riemannian connection is a solution of the Yang-Mills equations on the tangent bundle of $M$ (see [2]).

On the other hand, the notion of $k$-nullity distribution was introduced by A. Gray [12] and S. Tanno [22] in the study of Riemannian manifolds $(M, g)$, which is defined for any $p \in M$ as follows:

$$
N_{p}(k)=\left\{Z \in T_{p} M: R(X, Y) Z=k[g(Y, Z) X-g(X, Z) Y]\right\},
$$

where $X, Y$ denote arbitrary vectors in $T_{p} M$ and $k \in \mathbb{R}$. Later, D. E. Blair, T. Koufogiorgos and B. J. Papantoniou [6] introduced a generalized notion of the $k$-nullity distribution which is called the $(k, \mu)$-nullity distribution on contact metric manifolds $\left(M^{2 n+1}, \phi, \xi, \eta, g\right)$ and is defined for any $p \in M^{2 n+1}$ as follows:

$$
\begin{aligned}
N_{p}(k, \mu)=\left\{Z \in T_{p} M: R(X, Y) Z=\right. & k[g(Y, Z) X-g(X, Z) Y] \\
& +\mu[g(Y, Z) h X-g(X, Z) h Y]\},
\end{aligned}
$$

where $h=\frac{1}{2} \mathcal{L}_{\xi} \phi, \mathcal{L}$ denotes the Lie differentiation and $(k, \mu) \in \mathbb{R}^{2}$. Recently, G. Dileo and A. M. Pastore [11] introduced another generalized notion of the $k$-nullity distribution named the $(k, \mu)^{\prime}$-nullity distribution on almost Kenmotsu manifolds $\left(M^{2 n+1}, \phi, \xi, \eta, g\right)$, which is defined for any $p \in M^{2 n+1}$ as follows:

$$
\begin{aligned}
N_{p}(k, \mu)^{\prime}=\left\{Z \in T_{p} M: R(X, Y) Z=\right. & k[g(Y, Z) X-g(X, Z) Y] \\
& \left.+\mu\left[g(Y, Z) h^{\prime} X-g(X, Z) h^{\prime} Y\right]\right\},
\end{aligned}
$$

where $h=\frac{1}{2} \mathcal{L}_{\xi} \phi, h^{\prime}=h \circ \phi, \mathcal{L}$ denotes the Lie differentiation and $(k, \mu) \in \mathbb{R}^{2}$.

Almost Kenmotsu manifolds with $\xi$ belonging to the $k$-nullity and the $(k, \mu)^{\prime}-$ nullity distribution were studied in [21] and [11, 23, 24, 25] respectively. Contact metric manifolds with harmonic curvature tensor such that the characteristic vector field belongs to the $k$-nullity distribution were investigated by $\mathrm{C}$. Baikoussis and T. Koufogiougos in [2]. Generalizing the above result, K. Arslan, C. Murathan, C. Özgür and A. Yildiz [1] classified contact metric manifolds for which $\xi$ belongs to the $(k, \mu)$-nullity distribution and the curvature tensors are harmonic. We also refer the reader to B. J. Papantoniou [20] for some related results which extended the corresponding result shown in [2]. Motivated by these results mentioned above, the object of this paper is to study almost Kenmotsu manifolds with harmonic curvature tensors for which $\xi$ belongs to the $(k, \mu)^{\prime}$-nullity distribution. In fact, we mainly obtain the following classification theorem.

Theorem 1.1. Let $\left(M^{2 n+1}, \phi, \xi, \eta, g\right)$ be an almost Kenmotsu manifold of dimension $(2 n+1)$ such that the characteristic vector field $\xi$ belongs to the $(k, \mu)^{\prime}$-nullity distribution. Then the curvature tensor of $M^{2 n+1}$ is harmonic if and only if $M^{2 n+1}$ is locally isometric to either a Riemannian product $\mathbb{H}^{n+1}(-4) \times \mathbb{R}^{n}$ or an Einstein warped product $C \times{ }_{f} N^{2 n}$, where $C$ is an open interval with coordinate $t, N^{2 n}$ is a Ricci-flat almost Kähler manifold of dimension $2 n$ and the warping function is given by $f=c e^{t}$ for some positive constant $c$. 
The present paper is organized as follows. In Section 2, we first recall some well known basic formulas and properties of almost Kenmotsu manifolds. In Section 3, after introducing some key lemmas on almost Kenmotsu manifolds and some known properties on warped products, we finally present the detailed proof of our main theorem and some corollaries.

\section{Almost Kenmotsu manifolds}

We shall first recall some basic notions and properties of almost Kenmotsu manifolds. An almost contact structure (see Blair [5]) on a $(2 n+1)$-dimensional smooth manifold $M^{2 n+1}$ is a triplet $(\phi, \xi, \eta)$, where $\phi$ is a $(1,1)$-tensor field, $\xi$ a global vector field (which is called the characteristic vector field) and $\eta$ a 1 -form, such that

$$
\phi^{2}=-\mathrm{id}+\eta \otimes \xi, \quad \eta(\xi)=1,
$$

where id denotes the identity mapping. It is obvious to see from relation (2.1) that $\phi(\xi)=0, \eta \circ \phi=0$ and $\operatorname{rank}(\phi)=2 n$. A Riemannian metric $g$ on $M^{2 n+1}$ is said to be compatible with the almost contact structure $(\phi, \xi, \eta)$ if it satisfies

$$
g(\phi X, \phi Y)=g(X, Y)-\eta(X) \eta(Y)
$$

for any vector fields $X, Y$ on $M^{2 n+1}$. An almost contact structure endowed with a compatible Riemannian metric is said to be an almost contact metric structure (see Blair [5]). The fundamental 2-form $\Phi$ is defined by $\Phi(X, Y)=g(X, \phi Y)$ for any vector fields $X$ and $Y$ on $M^{2 n+1}$. An almost contact metric manifold such that $d \eta=0$ and $d \Phi=2 \eta \wedge \Phi$ is called an almost Kenmotsu manifold. It is known [5] that the normality of an almost contact structure is expressed by the vanishing of the tensor $N_{\phi}=[\phi, \phi]+2 d \eta \otimes \xi$, where $[\phi, \phi]$ is the Nijenhuis tensor of $\phi$. The normality of an almost Kenmotsu manifold is expressed by

$$
\left(\nabla_{X} \phi\right) Y=g(\phi X, Y) \xi-\eta(Y) \phi X
$$

for any vector fields $X, Y$. From Janssens and Vanhecke [13], a normal almost Kenmotsu manifold is said to be a Kenmotsu manifold.

We put $l=R(\cdot, \xi) \xi$ and $h=\frac{1}{2} \mathcal{L}_{\xi} \phi$ on an almost Kenmotsu manifold $\left(M^{2 n+1}, \phi, \xi, \eta, g\right)$, where $R$ is the curvature tensor of $M^{2 n+1}$ and $\mathcal{L}$ denotes the Lie differentiation. Thus, the two $(1,1)$-type tensor fields $l$ and $h$ are symmetric and satisfy

$$
h \xi=0, \quad l \xi=0, \operatorname{tr} h=0, \operatorname{tr}(h \phi)=0, \quad h \phi+\phi h=0 .
$$

We also have the following formulas presented in [10,11, 21]:

$$
\begin{gathered}
\nabla_{X} \xi=-\phi^{2} X-\phi h X, \\
\phi l \phi-l=2\left(h^{2}-\phi^{2}\right), \\
\operatorname{tr}(l)=S(\xi, \xi)=g(Q \xi, \xi)=-2 n-\operatorname{tr} h^{2}, \\
R(X, Y) \xi=\eta(X)(Y-\phi h Y)-\eta(Y)(X-\phi h X)+\left(\nabla_{Y} \phi h\right) X-\left(\nabla_{X} \phi h\right) Y,
\end{gathered}
$$


for any $X, Y \in \Gamma(T M)$, where $S, Q, \nabla$ and $\Gamma(T M)$ denote the Ricci tensor, the Ricci operator with respect to the metric $g$, the Levi-Civita connection of $g$ and the Lie algebra of all vector fields on $M^{2 n+1}$, respectively.

Finally, we recall that an almost contact metric manifold is said to be $\eta$-Einstein if the Ricci operator is given by

$$
Q=\alpha \mathrm{id}+\beta \eta \otimes \xi,
$$

where $\alpha$ and $\beta$ are both smooth functions on $M^{2 n+1}$.

\section{Harmonic curvature tensors}

We assume that $\left(M^{2 n+1}, \phi, \xi, \eta, g\right)$ is an almost Kenmotsu manifold for which $\xi$ belongs to the $(k, \mu)^{\prime}$-nullity distribution, then from (1.3) we have

$$
R(X, Y) \xi=k[\eta(Y) X-\eta(X) Y]+\mu\left[\eta(Y) h^{\prime} X-\eta(X) h^{\prime} Y\right]
$$

where $(k, \mu) \in \mathbb{R}^{2}$. Throughout the paper, we shall denote by $\mathcal{D}$ the distribution defined by $\mathcal{D}=\operatorname{ker}(\eta)=\operatorname{Im}(\phi)$. Replacing $Y$ by $\xi$ in relation (3.1) we obtain that $l X=k(X-\eta(X) \xi)+\mu h^{\prime} X$, thus, making use of (2.1) and (2.3) in this equation we get

$$
\phi l \phi X=-k(X-\eta(X) \xi)+\mu h^{\prime} X
$$

for any vector field $X$ on $M^{2 n+1}$. Substituting the above equation into (2.5) we have

$$
h^{\prime 2}=(k+1) \phi^{2}\left(\Leftrightarrow h^{2}=(k+1) \phi^{2}\right) .
$$

Letting $X \in \mathcal{D}$ be the eigenvector field of $h^{\prime}$ corresponding to the eigenvalue $\lambda$, thus from (3.2) it is easy to see that $\lambda^{2}=-(k+1)$ is a constant. It follows from relation (3.2) that $k \leq-1$ and $\lambda= \pm \sqrt{-k-1}$. Clearly, $h^{\prime} \neq 0$ if and only if $k<-1$. In case of $k<-1$, we denote the eigenspaces corresponding to the eigenvalues $\lambda$ and $-\lambda$ of $h^{\prime}$ on $\mathcal{D}$ by $[\lambda]^{\prime}$ and $[-\lambda]^{\prime}$ respectively, where $\lambda \neq 0$.

Lemma 3.1 (Proposition 4.2 of [11]). Let $\left(M^{2 n+1}, \phi, \xi, \eta, g\right)$ be an almost Kenmotsu manifold with $\xi$ belonging to the $(k, \mu)^{\prime}$-nullity distribution. If $h^{\prime} \neq 0$, then for any $X_{\lambda}, Y_{\lambda}, Z_{\lambda} \in[\lambda]^{\prime}$ and $X_{-\lambda}, Y_{-\lambda}, Z_{-\lambda} \in[-\lambda]^{\prime}$, the curvature tensor satisfies:

$$
\begin{aligned}
R\left(X_{\lambda}, Y_{\lambda}\right) Z_{-\lambda} & =0 \\
R\left(X_{-\lambda}, Y_{-\lambda}\right) Z_{\lambda} & =0 \\
R\left(X_{\lambda}, Y_{-\lambda}\right) Z_{\lambda} & =(k+2) g\left(X_{\lambda}, Z_{\lambda}\right) Y_{-\lambda} \\
R\left(X_{\lambda}, Y_{-\lambda}\right) Z_{-\lambda} & =-(k+2) g\left(Y_{-\lambda}, Z_{-\lambda}\right) X_{\lambda} \\
R\left(X_{\lambda}, Y_{\lambda}\right) Z_{\lambda} & =(k-2 \lambda)\left[g\left(Y_{\lambda}, Z_{\lambda}\right) X_{\lambda}-g\left(X_{\lambda}, Z_{\lambda}\right) Y_{\lambda}\right] \\
R\left(X_{-\lambda}, Y_{-\lambda}\right) Z_{-\lambda} & =(k+2 \lambda)\left[g\left(Y_{-\lambda}, Z_{-\lambda}\right) X_{-\lambda}-g\left(X_{-\lambda}, Z_{-\lambda}\right) Y_{-\lambda}\right]
\end{aligned}
$$

and $\mu=-2$.

Making use of the curvature properties shown by Lemma 3.1, the present authors obtained the following result by a straightforward computation. 
Lemma 3.2 (Lemma 3.2 of [23]). Let $\left(M^{2 n+1}, \phi, \xi, \eta, g\right)$ be an almost Kenmotsu manifold with $\xi$ belonging to the $(k, \mu)^{\prime}$-nullity distribution. If $h^{\prime} \neq 0$, then the Ricci operator $Q$ of $M^{2 n+1}$ is given by

$$
Q=-2 n i d+2 n(k+1) \eta \otimes \xi-2 n h^{\prime}
$$

Moreover, the scalar curvature of $M^{2 n+1}$ is $2 n(k-2 n)$.

Next, by using the Ricci operator shown by the above lemma, we obtain the following result to prove our main theorem.

Lemma 3.3. Let $\left(M^{2 n+1}, \phi, \xi, \eta, g\right)$ be an almost Kenmotsu manifold with $\xi$ belonging to the $(k, \mu)^{\prime}$-nullity distribution. If $h^{\prime} \neq 0$, then

$$
\begin{aligned}
& \left(\nabla_{X} h^{\prime}\right) Y=\left(\nabla_{Y} h^{\prime}\right) X ; \\
& \left(\nabla_{\xi} Q\right) X=-2 n\left(\nabla_{\xi} h^{\prime}\right) X ; \\
& \left(\nabla_{X} Q\right) \xi=2 n\left((k+1) X+(k+2) h^{\prime} X+h^{\prime 2} X\right)
\end{aligned}
$$

for any vector fields $X, Y$ on the distribution $\mathcal{D}$.

Proof. Letting $X, Y \in \mathcal{D}$ in (3.1) yields that $R(X, Y) \xi=0$. Thus, relation (3.10) follows from (2.7) for any $X, Y \in \mathcal{D}$. Making use of (2.4) and taking the covariant differentiation of (3.9) along arbitrary vector field $Y \in \Gamma(T M)$, we obtain

$$
\begin{aligned}
& \left(\nabla_{Y} Q\right) X+2 n\left(\nabla_{Y} h^{\prime}\right) X \\
= & 2 n(k+1)\left[\eta(X) Y-2 \eta(X) \eta(Y) \xi+\eta(X) h^{\prime} Y+g(X, Y) \xi+g\left(h^{\prime} X, Y\right) \xi\right]
\end{aligned}
$$

for any $X, Y \in \Gamma(T M)$. Next, letting $Y \in \mathcal{D}$ and $X=\xi$ in (3.13) and making use of (2.4), we obtain relation (3.12). Similarly, letting $Y=\xi$ and $X \in \mathcal{D}$ in (3.13) we get (3.11).

The following key lemma is also useful in the proof of our main theorem.

Lemma 3.4 (Lemma 4 of [7]). Let $C$ be an interval of $\mathbb{R}$, considered with its standard metric, $f: C \rightarrow \mathbb{R}$ a non-constant positive $C^{\infty}$-function and $N$ an 2 -dimensional Riemannian manifold, $n \geq 1$, Then the following conditions are equivalent:

(1) $C \times{ }_{f} N$ has harmonic Riemannian curvature tensor;

(2) $N$ is an Einstein manifold and the positive function $\varphi=f^{\frac{2 n+1}{4}}: C \rightarrow \mathbb{R}$ satisfies the following ordinary differential equation

$$
\varphi^{\prime \prime}-\frac{(2 n+1) r}{8 n} \varphi^{\frac{2 n-3}{2 n+1}}=p \varphi
$$

for some real number $p, r$ being the constant scalar curvature of $N$.

Finally, we may present the detailed proof of Theorem 1.1 as follows.

Proof of Theorem 1.1. Suppose that $\left(M^{2 n+1}, \phi, \xi, \eta, g\right)$ is an almost Kenmotsu manifold with $\xi$ belonging to the $(k, \mu)^{\prime}$-nullity distribution. By Dileo and Pastore 
[11], it follows that $k \leq-1$. Therefore we may separate our discussions into two case as follows.

Case 1: $k<-1$. By Proposition 4.1 of [11], the assumption $k<-1$ is equivalent to $h^{\prime} \neq 0$, then Lemma 3.1, 3.2 and 3.3 are applicable in this context. Letting $X, Y \in \mathcal{D}$ in relation (3.13) and making use of (3.10) we have that $\left(\nabla_{X} Q\right) Y=$ $\left(\nabla_{Y} Q\right) X$ for any $X, Y \in \mathcal{D}$. By a direct calculation we may see that the curvature tensor of $M^{2 n+1}$ is harmonic if and only if $\left(\nabla_{X} Q\right) \xi=\left(\nabla_{\xi} Q\right) X$ for any $X \in \mathcal{D}$. Therefore, in view of equations (3.11) and (3.12) we know that the curvature tensor $R$ is harmonic if and only if

$$
\left(\nabla_{\xi} h^{\prime}\right) X+(k+1) X+(k+2) h^{\prime} X+h^{\prime 2} X=0
$$

for any $X \in \mathcal{D}$. Next, letting $X \in[\lambda]^{\prime}$ in (3.15) and taking the inner product with $X$ on both sides of (3.15) we obtain

$$
\lambda(k+2)=0,
$$

where we have used the identity $g\left(\left(\nabla_{\xi} h^{\prime}\right) X, X\right)=\xi(\lambda) g(X, X)=0$ (noticing that $\lambda$ is a constant). In view of $h^{\prime} \neq 0$, we know that $\lambda$ is non-vanishing and hence it follows from (3.16) that $k=-2$ and hence $\lambda= \pm 1$. The following proof is similar to that of the corresponding results in [11, 24, 25]. Without losing the generality we now choose $\lambda=1$, then relations (3.7) and (3.8) can be re-written respectively as follows:

$$
R\left(X_{\lambda}, Y_{\lambda}\right) Z_{\lambda}=-4\left[g\left(Y_{\lambda}, Z_{\lambda}\right) X_{\lambda}-g\left(X_{\lambda}, Z_{\lambda}\right) Y_{\lambda}\right]
$$

and

$$
R\left(X_{-\lambda}, Y_{-\lambda}\right) Z_{-\lambda}=0
$$

for any $X_{\lambda}, Y_{\lambda}, Z_{\lambda} \in[\lambda]^{\prime}$ and $X_{-\lambda}, Y_{-\lambda}, Z_{-\lambda} \in[-\lambda]^{\prime}$. On the other hand, noticing that $\mu=-2$ then it follows from (3.1) that $K(X, \xi)=-4$ for any $X \in[\lambda]^{\prime}$ and $K(X, \xi)=0$ for any $X \in[-\lambda]^{\prime}$. As shown in [11], the distribution $[\xi] \oplus[\lambda]^{\prime}$ is integrable with totally geodesic leaves immersed in $M^{2 n+1}$, and the distribution $[-\lambda]^{\prime}$ is integrable with totally umbilical leaves immersed in $M^{2 n+1}$ whose mean curvature vector field is given by $H=-(1-\lambda) \xi$. Taking into account $\lambda=1$, we know that both the two orthogonal distribution $[\xi] \oplus[\lambda]^{\prime}$ and $[-\lambda]$ are integrable with totally geodesic leaves immersed in $M^{2 n+1}$. Therefore, $M^{2 n+1}$ is locally isometric to a Riemannian product of an $(n+1)$-dimensional manifold of constant sectional curvature -4 and a flat $n$-dimensional manifold.

Conversely, if $M^{2 n+1}$ is locally isometric to the product $\mathbb{H}^{n+1}(-4) \times \mathbb{R}^{n}$, we observe from Proposition 4.2 of [3] or Remark 4.1 of [11] that such a product is locally symmetric, i.e., $\nabla R=0$, this implies that the Ricci tensor is symmetric (i.e., $\nabla S=0$ ) and hence the Riemannian curvature tensor is harmonic.

Case 2: $k=-1$. Suppose that $\left(M^{2 n+1}, \phi, \xi, \eta, g\right)$ is an almost Kenmotsu manifold with the characteristic vector field $\xi$ belonging to the $(k, \mu)^{\prime}$-nullity distribution. From [11, 21], it follows that the following three conditions are equivalent: (i) $h=0$; (ii) $k=-1$; (iii) the Reeb foliation of $M^{2 n+1}$ is conformal. By Theorem 2 of [10], the vanishing of $h$ implies that $M^{2 n+1}$ is locally isometric to a warped product $C \times{ }_{f} N^{2 n}$, where $C$ is an open interval with coordinate $t, N^{2 n}$ is an almost 
Kähler manifold of dimension $2 n$ and the warping function is given by $f=c e^{t}$ for some positive constant $c$.

Since $M^{2 n+1}$ has harmonic curvature tensor, by applying Lemma 3.4 we see that $N^{2 n}$ is an Einstein manifold. Thus, we may assume $S^{N}=\gamma g_{N}$ for certain smooth function $\gamma$ on $N^{2 n}$, where $S^{N}$ denotes the Ricci tensor of $N^{2 n}$ with respect to the Riemannian metric $g^{N}$ of $N^{2 n}$. In particular, making use of $f=c e^{t}$ in (3.14) yields that $r=0$ and hence the Ricci tensor of $N^{2 n}$ vanishes, this means that $N^{2 n}$ is a Ricci-flat almost Kähler manifold.

We also observe from the third term of Corollary 43 of [19] that the Ricci tensor of the Riemanian warped product $\left(C \times{ }_{f} N^{2 n}, g\right)$ is given as follows:

$$
S(V, W)=S^{N}(V, W)-g(V, W) f^{\sharp}
$$

for any vector fields $V, W$ on the Riemannian fiber $\left(N^{2 n}, g^{N}\right)$, where $S$ and $S^{N}$ denote the Ricci tensors of Riemannian manifolds $\left(C \times{ }_{f} N^{2 n}, g\right)$ and $\left(N^{2 n}, g^{N}\right)$ respectively, and

$$
f^{\sharp}=\frac{\Delta f}{f}+\left(\operatorname{dim}\left(N^{2 n}\right)-1\right) \frac{\langle\operatorname{grad} f, \operatorname{grad} f\rangle}{f^{2}} .
$$

Making use of $f=c e^{t}$ in the above equation we obtain $f^{\sharp}=2 n$. Putting this relation into (3.17) and noticing that the Ricci tensor of $N^{2 n}$ vanishes, we obtain $S(V, W)=-2 n g(V, W)$ for any vector fields $V, W$ on $N^{2 n}$. Using $h=0$ in (2.7) we have $R(X, Y) \xi=-\eta(Y) X+\eta(X) Y$ for any vector fields $X, Y$ on $M^{2 n+1}$, hence, by a direct calculation we get $S(\xi, \xi)=-2 n g(\xi, \xi)=-2 n$. Moreover, we obtain from the second term of Corollary 43 of [19] that $S(\xi, V)=S(V, \xi)=0$ for any vector fields $V$ on $N^{2 n}$. Therefore, by the above arguments we know that the warped product $C \times{ }_{f} N^{2 n}$ is an Einstein manifold with the Ricci operator $Q=-2 n i d$. It is easy to check that the converse is also true. This completes the proof.

Some results concerning Einstein warped products were shown in [15] and [18]. Notice that in our case the Einstein warped product $C \times_{f} N^{2 n}$ is noncompact. In fact, making use of $h=0(\Leftrightarrow k=-1)$ in (2.4) we obtain that the divergence of $\xi$ is given by $\operatorname{div} \xi=2 n$.

Corollary 3.1. The curvature tensor of an almost Kenmotsu manifold with conformal foliation is harmonic if and only if the manifold is Einstein.

By Proposition 2 of [10], we see that an almost Kenmotsu manifolds is Kenmotsu if and only if the integral manifolds of the distribution $\mathcal{D}$ are Kählerian and the tensor field $h$ vanishes. Therefore, Corollary 3.1 follows from Case 2 of Theorem 1.1 and can be regarded as a generalization of Theorem 2 of [4].

Let $M^{2 n+1}$ be an $\eta$-Einstein almost Kenmotsu manifold (i.e., $Q=\alpha \mathrm{id}+\beta \eta \otimes \xi$ ) with conformal Reeb foliation and $\beta$ a constant, A. M. Pastore and V. Saltarelli in [21] proved that $M^{2 n+1}$ is an Einstein manifold with the Ricci operator $Q=-2$ nid.

Corollary 3.2. Let $\left(M^{2 n+1}, \phi, \xi, \eta, g\right)$ be an almost Kenmotsu manifold such that the characteristic vector field $\xi$ belongs to the $(k, \mu)^{\prime}$-nullity distribution with $k<-1$. Then 
the curvature tensor of $M^{2 n+1}$ is harmonic if and only if $M^{2 n+1}$ is locally isometric to $\mathbb{H}^{n+1}(-4) \times \mathbb{R}^{n}$.

The above corollary follows directly from Case 1 of Theorem 1.1. It is well known that local symmetry condition implies Ricci symmetry, therefore, Corollary 3.2 extends one conclusion of Proposition 4.1 of [11]. It is also known that the Ricci symmetry condition implies that the curvature tensor is harmonic, hence Corollary 3.1 is also a generalization of Corollary 4.3 of [24].

It is well known that the Weyl conformal curvature tensor $C$ is defined on a Riemanian manifold $(M, g)$ of dimension $m>3$ as follows:

$$
\begin{aligned}
& C(X, Y) Z \\
= & R(X, Y) Z+\frac{r}{(m-1)(m-2)}\{g(Y, Z) X-g(X, Z) Y\} \\
& -\frac{1}{m-2}\{S(Y, Z) X-S(X, Z) Y+g(Y, Z) Q X-g(X, Z) Q Y\}
\end{aligned}
$$

for any vector fields $X, Y, Z$ on $M$, where $r$ denotes the scalar curvature of $M$. Notice that the Weyl conformal curvature tensor $C$ is said to be harmonic if $\operatorname{div} C=0$. For a Riemanian manifold $M$ of dimension $m>3$, a straightforward calculation gives that the Weyl curvature tensor is harmonic if and only if

$$
\left(\nabla_{X} S\right)(Y, Z)-\left(\nabla_{Y} S\right)(X, Z)=\frac{1}{2(m-1)}\{X(r) g(Y, Z)-Y(r) g(X, Z)\}
$$

for any vector fields $X, Y, Z$ on $M$. Assume that $M^{2 n+1}$ is an almost Kenmotsu manifold with the characteristic vector field $\xi$ belonging to the $(k, \mu)^{\prime}$-nullity distribution and $k<-1$, by Lemma 3.2 we see that the scalar curvature of $M^{2 n+1}$ is a constant. Consequently, the following result directly follows from Theorem 1.1 and relation (3.19).

Corollary 3.3. Let $\left(M^{2 n+1}, \phi, \xi, \eta, g\right)$ be an almost Kenmotsu manifold of dimension $>3$ such that the characteristic vector field $\xi$ belongs to the $(k, \mu)^{\prime}$-nullity distribution and $k<-1$. Then the Weyl conformal curvature tensor of $M^{2 n+1}$ is harmonic if and only if $M^{2 n+1}$ is locally isometric to $\mathbb{H}^{n+1}(-4) \times \mathbb{R}^{n}$.

\section{Acknowledgements}

This project is supported by Natural Science Foundation of China (No. 11371076) and the Fundamental Research Funds for the Central Universities (No. DUT14ZD208). The authors would like to thank the referee very much for his or her some valuable suggestions which have improved this paper. 


\section{References}

[1] K. Arslan, C. Murathan, C. Özgür and A. Yildiz, On contact metric R-harmonic manifolds, Balkan J. Geom. Appl. 5 (2000), 1-6.

[2] C. Baikoussis and T. Koufogiougos, On a type of contact manifolds, J. Geom. 46 (1993), 1-9.

[3] M. Bertola and D. Gouthier, Lie triple system and warped products, Rend. Mat. Appl. VII Ser. 21 (2001), 275-293.

[4] T. Q. Binh, L. Tamássy, U. C. De and M. Tarafdar, Some remarks on almost Kenmotsu manifolds, Math. Pannon. 13(1) (2002), 31-39.

[5] D. E. Blair, Riemannian geometry of contact and symplectic manifolds, Progress in Mathematics, Volume 203, Birkhäuser, 2010.

[6] D. E. Blair, T. Koufogiorgos and B.J. Papantoniou, Contact metric manifolds satisfying a nullity condition, Israel J. Math. 91 (1995), 189-214.

[7] A. Derdziński, Classification of certain compact Riemannian manifolds with harmonic curvature and non-parallel Ricci tensor, Math. Z. 172 (1980), 273-280.

[8] A. Derdziński, On compact Riemannian manifolds with harmonic curvature, Math. Ann. 259 (1982), 145-152.

[9] A. Derdzinski and W. Roter, Some theorems on conformally symmetric manifolds, Tensor (N.S.) 32 (1978), 11-23.

[10] G. Dileo and A. M. Pastore, Almost Kenmotsu manifolds and local symmetry, Bull. Belg. Math. Soc. Simon Stevin 14 (2007), 343-354.

[11] G. Dileo and A. M. Pastore, Almost Kenmotsu manifolds and nullity distributions, J. Geom. 93 (2009), 46-61.

[12] A. Gray, Spaces of contancy of curvature operators, Proc. Amer. Math. Soc. 17 (1966), 897-902.

[13] D. Janssens and L. Vanhecke, Almost contact structures and curvature tensors, Kodai Math. J. 4 (1981), 1-27.

[14] K. Kenmotsu, A class of almost contact Riemannian manifolds, Tôhoku Math. J. 24 (1972), 93-103.

[15] D. S. Kim and Y. H. Kim, Compact Einstein warped products spaces with nonpositive scalar curvature, Proc. Amer. Math. Soc. 131 (2003), 2573-2576.

[16] M. Memerthzheim and H. Reckziegel, Hypersurface with harmonic curvature in space of constant curvature, Cologne, March 1993.

[17] S. Mukhopadhyay and B. Barua, On a type of non-flat Riemannian manifold, Tensor 56 (1995), 227-232. 
[18] M. T. Mustafa, A non-existence result for compact Einstein warped products, J. Phys. A: Math. Gen. 38 (2005), L791-L793.

[19] B. O'Neill, Semi-Riemannian geometry with application to relativity, Academic Press, New York, 1983.

[20] B. J. Papantoniou, Contact manifolds, harmonic curvature tensor and $(k, \mu)$ nullity distribution, Comment. Math. Univ. Carolin. 34(2) (1993), 323-334.

[21] A. M. Pastore and V. Saltarelli, Almost Kenmotsu manifolds with conformal Reeb foliation, Bull. Belg. Math. Soc. Simon Stevin 21 (2012), 343-354.

[22] S. Tanno, Some differential equations on Riemannian manifolds, J. Math. Soc. Japan 30 (1978), 509-531.

[23] Y. Wang and X. Liu, On almost Kenmotsu manifolds satisfying some nullity distributions, submitted.

[24] Y. Wang and X. Liu, Second order parallel tensors on almost Kenmotsu manifolds satisfying the nullity distributions, Filomat 28(4) (2014), 839-847.

[25] Y. Wang and X. Liu, Riemannian semisymmetric almost Kenmotsu manifolds and nullity distributions, Ann. Polon. Math. 112(1) (2014), 37-46.

College of Mathematics and Information Science

Henan Normal University

Xinxiang 453007, Henan, P. R. China

E-mail: wyn051@163.com

School of Mathematical Sciences

Dalian University of Technology

Dalian 116024, Liaoning, P. R. China

E-mail: ximinliu@dlut.edu.cn 